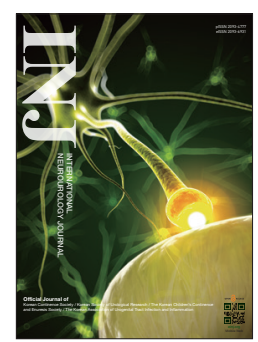

\title{
ADRB3, ROCK2, and GEF Levels in Overactive Bladder Patients
}

\author{
Elif Firat ${ }^{1}$, Zafer Aybek ${ }^{2}$, Hülya Aybek ${ }^{1}$ \\ ${ }^{1}$ Department of Medical Biochemistry, Pamukkale University School of Medicine, Denizli, Turkey \\ ${ }^{2}$ Department of Urology, Pamukkale University School of Medicine, Denizli, Turkey
}

Purpose: The aim of this study was to evaluate changes in levels of adrenergic receptor $\beta 3$ (ADRB3), Rho-related kinase 2 (ROCK2), and guanine nucleotide exchange factor (GEF), which play key roles in the adrenergic and cholinergic pathways of contraction-relaxation harmony in voiding physiology, and to explore the relationship between these proteins and overactive bladder (OAB).

Methods: This study included 60 idiopathic $\mathrm{OAB}$ patients and a healthy control group. A validated OAB-validated 8 questionnaire was completed by all participants. Serum levels of ADRB3, ROCK2, and GEF were examined with enzyme-linked immunosorbent assays. Patient and control groups were compared in terms of these levels, and receiver operating characteristic (ROC) curves were generated for all parameters.

Results: The levels of ROCK2 were significantly elevated, but there were no correlations between the OAB symptom score and the serum levels of ROCK2, ADRB3, and GEF in OAB patients. In the ROC analysis, ROCK2 alone provided the strongest potential relationship (area under the curve $=0.651$ ) with $84.9 \%$ sensitivity. The ROCK2+GEF combination provided a satisfactory relationship (AUC $=0.755$ ). The AUC for the ADRB3+ROCK2+GEF combination was 0.752 , with $64.2 \%$ sensitivity and $88.2 \%$ specificity.

Conclusions: The study results suggest that alterations in serum ROCK2 levels and the use of this parameter in combination with ADRB3 and GEF levels can shed light on the pathophysiology of idiopathic OAB syndrome and provide a new perspective for treatment.

Keywords: Adrenergic receptor $\beta 3$; Biomarkers; Guanine nucleotide exchange factor; Overactive bladder; Rho-related kinase

- Funding Support: This study was supported by the Pamukkale University Scientific Research Projects Department (grant number: 2019HZDP009).

- Research Ethics: This study was approved by the Pamukkale University Non-Interventional Clinical Research Ethics Committee (reference number: 03; Date: 05.02.2019) and informed consent was obtained from all individuals included in the study.

- Conflict of Interest: No potential conflict of interest relevant to this article was reported.

\section{- HIGHLIGHTS}

- Serum ROCK2 levels were significantly elevated in OAB patients. Could ROCK2 be a new treatment target?

- Receiver operating characteristic analysis suggested that ROCK2, alone and especially in combination with GEF or ADRB3, could act as an auxiliary biomarker for the diagnosis of OAB.

\section{INTRODUCTION}

Overactive bladder $(\mathrm{OAB})$ is a symptom syndrome consisting of urgency, with or without urge incontinence, usually with frequency and nocturia. For diagnosis, the presence of infection or an obvious pathology should be ruled out. OAB is a clinical
Corresponding author: Elif Frrat (iD https://orcid.org/0000-0001-8394-3810 Department of Medical Biochemistry, Pamukkale University School of Medicine, Pamukkale Universitesi Tip Fakultesi Hastanesi, Merkez Laboratuvari, Tibbi Biyokimya Anabilim Dali, Denizli, Turkye Email: elifbasak43@hotmail.com

Submitted: February 2, 2021 / Accepted after revision: March 29, 2021 
diagnosis [1]. The assessment of $\mathrm{OAB}$ is very important, especially for the evaluation of treatment effectiveness. $O A B$ is diagnosed by symptoms only, and symptoms are subjective definitions that vary from person to person. Recent studies have proposed using several substances such as urinary proteins as auxiliary parameters for $\mathrm{OAB}$, but there is a lack of studies comparing these markers, and these proteins have been shown to have low sensitivity and specificity [2].

The molecular mechanisms underlying the clinical status of $\mathrm{OAB}$ are still not fully understood. Although the exact mechanism of $\mathrm{OAB}$ has not been elucidated, the most widely accepted theory of its pathogenesis is detrusor muscle hyperactivity because of increased contractions or decreased relaxation in the detrusor smooth muscle [3].

The parasympathetic cholinergic system is responsible for contractions in the bladder muscle. Agonists activate Rho through the activation of muscarinic receptor M3 in smooth muscle [4]. Rho activity is regulated by guanine nucleotide exchange factor (GEF), which regulates the separation of GDP from Rho and the binding of GTP [5]. GTP-dependent Rho activates Rho-related kinase 2 (ROCK2), which then phosphorylates myosin phosphatase and inhibits myosin phosphatase activity. ROCK2 also phosphorylates myosin light chain (MLC) directly. Thus, ROCK2 may be able to regulate smooth muscle contraction via 2 processes: the inactivation of myosin phosphatase and direct MLC phosphorylation. Increased GEF levels in smooth muscle cause excess ROCK2 expression, and increased ROCK2 activity causes an increase in the phosphorylation of MLC. This increases contraction and can cause $\mathrm{OAB}$ syndrome $[4,6,7]$. In recent years, increasingly many studies have explored the vital role of ROCK2 in many diseases, especially vascular and neurodegenerative diseases. According to these developments, treatments targeting ROCK2 and the use of ROCK2 inhibitors in the treatment of many diseases are on the agenda [8].

The sympathetic system relaxes the bladder smooth muscle via adrenergic receptor $\beta 3$ (ADRB3). This receptor plays an important role in many tissues, such as smooth muscle, and its relaxation effect is exerted through the stimulation of adenyl cyclase and the accumulation of cAMP [4]. A decrease in ADRB3 levels may be responsible for reduced relaxation via hypofunction of the receptor in the pathophysiology of $\mathrm{OAB}$ [9].

In line with these pathophysiological theories, the pharmacological therapy used in $\mathrm{OAB}$ patients consists of antimuscarinic agents or beta-3 adrenoceptor agonists $[10,11]$. However, no consensus exists regarding the difference between beta-adren- ergic therapy and antimuscarinic therapy in terms of effectiveness [12]. Nonetheless, the serious adverse effects of antimuscarinic agents make it difficult for patients to continue treatment. Therefore, several questions arise: Which patients should receive antimuscarinic treatment, and which should receive betaadrenergic treatment? Which pharmacological treatment will be more successful in which patient? Could there be other pharmacological treatment targets for these patients?

The aim of this study was to investigate whether ADRB3, ROCK2, and GEF levels shed light on the molecular mechanisms of OAB. Furthermore, deciding which treatment is appropriate for which patient at the time of diagnosis - thereby enabling the choice of a patient-specific treatment - will be an important step in increasing the success of $\mathrm{OAB}$ management. If changes in levels of these parameters affect the pathogenesis of $\mathrm{OAB}$, the use of biomarkers may be considered to identify different $\mathrm{OAB}$ phenotypes, which can be the target of new treatment options.

\section{MATERIALS AND METHODS}

\section{Selection of Patients and Healthy Volunteers}

The inclusion of at least 102 participants (51 patients and 51 controls) would result in $80 \%$ power with a $95 \%$ confidence level according to the power analysis performed before the study. Sixty female OAB patients aged $>18$ years were included in the study. Patients who had troubling urgency or a compelling desire to void and also had a score of at least 11 points on the OAB-validated $8(\mathrm{OAB}-\mathrm{V} 8)$ questionnaire were diagnosed as having $\mathrm{OAB}$. They had not previously received any treatment for $\mathrm{OAB}$. The exclusion criteria were having any lower urinary tract dysfunction, urinary tract diseases such as stones, neurogenic bladder, bladder obstruction, tumors, infections, severe renal failure, liver failure, and myasthenia gravis. Sixty healthy individuals, who were matched to the patients in terms of age, sex, body weight, height, and body mass index (BMI), comprised a control group. They were selected as volunteers from patients' relatives and hospital staff who did not have any urinary complaints or other diseases. A validated Turkish version of the OAB-V8 questionnaire was completed by all participants [13].

\section{Measurement of Serum ADRB3, GEF, and ROCK2 Levels}

Whole blood samples were collected from participants in the morning after at least 8 hours of fasting. Serum samples from 
whole blood were centrifuged after coagulation and then stored at $-80^{\circ} \mathrm{C}$ until analysis. Enzyme-linked immunosorbent assay (ELISA) analysis was performed using an ELx800 Absorbance Microplate Reader (BioTek Instruments, Winooski, VT, USA) detection system using a human ADRB3 ELISA Kit (SinoGeneClon Biotech, Hangzhou, China, Cat. No: SG-16339), a human GEF ELISA kit (SinoGeneClon Biotech, China, Cat. No: SG16405), and a human ROCK2 ELISA Kit (SinoGeneClon Biotech, China, Cat. No: SG-10439) following the manufacturer's protocol with sensitivity of $0.1 \mathrm{ng} / \mathrm{mL}, 0.1 \mathrm{ng} / \mathrm{mL}$, and $2 \mathrm{pg} / \mathrm{mL}$, respectively. The intra-assay and inter-assay coefficients of variation of all 3 kits were $<8 \%$ and $<10 \%$, respectively. The results were analyzed using a Gen5 Microplate Reader and Imaging Software (BioTek Instruments, Winooski, VT, USA).

\section{Statistical Analysis}

Data were analyzed statistically using IBM SPSS Statistics ver. 25.0 (IBM Co., Armonk, NY, USA). Descriptive statistics and the nonparametric Mann-Whitney U-test were used. The correlations between $\mathrm{OAB}$ symptom scores and expression levels of proteins were done by calculating Spearman correlation coefficients. Receiver operating characteristic (ROC) curves were created to evaluate potential relationships between the diagnosis of $\mathrm{OAB}$ and variables by calculating the area under the curve (AUC) and to evaluate their contribution to the diagnosis by calculating sensitivity and specificity values. The combinations established using stepwise logistic regression analysis were evaluated with ROC curves. The P-value for statistical significance was considered as $<0.05$.

\section{RESULTS}

There was no significant difference between the patients and controls in terms of mean age, body weight, height, and BMI (Table 1).

Although the analysis was intended to include 60 patients and 60 control subjects, some serum samples did not react during the analysis; therefore, in the patient and control group a different number of samples were analyzed for each protein (Table 2). Nonetheless, the study was continued, as the number

Table 1. Age, height, body weight, and BMI values in patient and control groups

\begin{tabular}{lllll}
\hline Variable & Group & Mean \pm SD & Median (range) & P-value \\
\hline Age $(\mathrm{yr})$ & Healthy & $55.8 \pm 11.8$ & $58.5(27.0-76.0)$ & 0.483 \\
& OAB & $56.6 \pm 15.1$ & $60.0(23.0-83.0)$ & 0.080 \\
Height $(\mathrm{m})$ & Healthy & $1.59 \pm 0.07$ & $1.60(1.40-1.72)$ & $0.56(1.39-1.70)$ \\
& OAB & $1.57 \pm 0.64$ & $70.0(50.0-112.0)$ & $72.8(40.0-125.0)$ \\
Body weight $(\mathrm{kg})$ & Healthy & $72.0 \pm 13.0$ & $28.1(19.2-43.8)$ & 0.101 \\
& OAB & $73.9 \pm 16.4$ & $30.3(17.8-45.9)$ & $28.6 \pm 5.05$ \\
\hline
\end{tabular}

BMI, body mass index; SD, standard deviation; OAB, overactive bladder.

Table 2. Comparison of intergroup ADRB3, ROCK2, and GEF levels

\begin{tabular}{lrcr}
\hline Variable & \multicolumn{1}{c}{ Group } & Median (range) & P-value \\
\hline ADRB3 $(\mathrm{ng} / \mathrm{mL})$ & Healthy $(\mathrm{n}=58)$ & $13.88(4.36-189.90)$ & 0.996 \\
& OAB $(\mathrm{n}=58)$ & $14.87(5.72-88.47)$ & $0.003^{*}$ \\
ROCK2 $(\mathrm{pg} / \mathrm{mL})$ & Healthy $(\mathrm{n}=56)$ & $119.60(6.78-1,532.00)$ & $225.30(73.96-1,461.00)$ \\
GEF $(\mathrm{ng} / \mathrm{mL})$ & OAB $(\mathrm{n}=58)$ & $7.40(0.16-79.89)$ & 0.451 \\
& Healthy $(\mathrm{n}=54)$ & $6.52(0.24-35.52)$ & \\
\hline
\end{tabular}

ADRB3, adrenergic receptor $\beta 3$; ROCK2, Rho-related kinase 2; GEF, guanine nucleotide exchange factor; OAB: Overactive bladder. ${ }^{\star} \mathrm{P}<0.05$, statistically significant difference. 
of serum samples included in the final analysis satisfied the number of participants indicated by power analysis.

Serum levels of ROCK2 were significantly higher in the OAB patients (median, $225.25 \mathrm{pg} / \mathrm{mL}$; range, 73.96-1,461.73 pg/mL) than in the control subjects (median, $119.61 \mathrm{pg} / \mathrm{mL}$; range, 6.78-1,532.16 pg/mL) $(\mathrm{P}=0.003)$. OAB subjects also tended to show higher serum levels of ADRB3 and lower GEF levels than those in the control group, but neither comparison showed a statistically significant difference (Table 2 ). There was no correlation between protein levels and the OAB symptom score obtained from the $\mathrm{OAB}-\mathrm{V} 8$ questionnaire.

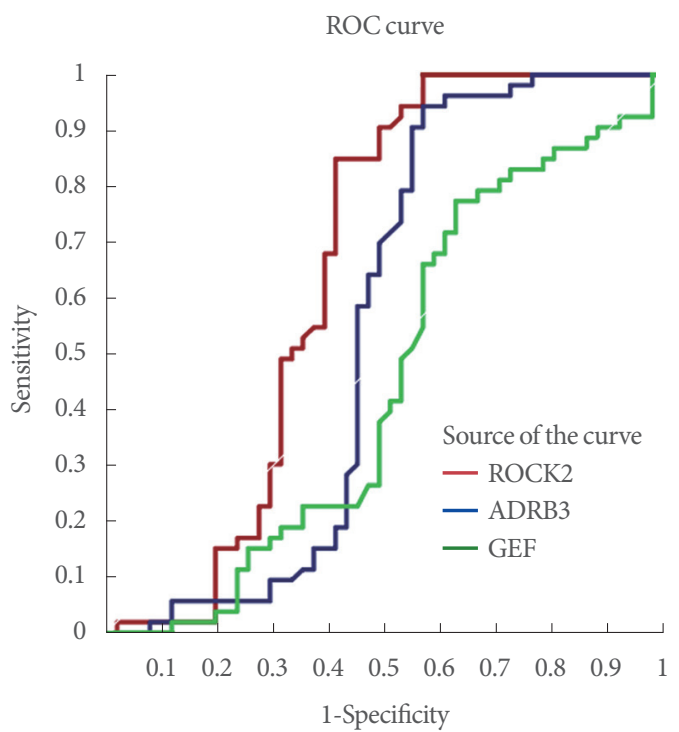

In the ROC curve (Fig. 1A), serum ROCK2 levels showed a significant relationship. The sensitivity and specificity of ROCK2 were $84.9 \%$ and $58.8 \%$ (95\% confidence interval [CI], 0.5350.767; $\mathrm{P}=0.008$ ), respectively, with an AUC of 0.651. In the ROC curves of serum ADRB3 and GEF alone, no significant results were found. ROC curves were also obtained through stepwise logistic regression by creating double and triple combinations of these 3 parameters. Some combinations showed stronger relationships with $\mathrm{OAB}$ than ROCK2 alone. The ADRB3 + ROCK2 combination provided $69 \%$ sensitivity and $70.4 \%$ specificity $(95 \%$ CI, $0.602-0.798 ; \mathrm{P}<0.001)$ with an AUC

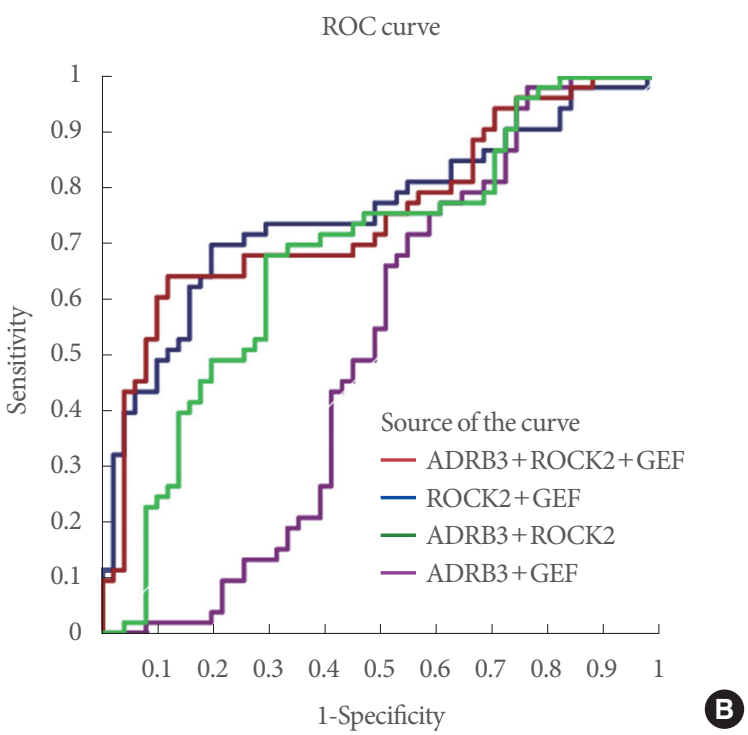

Fig. 1. ROC curves comparing the potential relationships for each (A) and combinations (B) of ROCK2, ADRB3, and GEF in OAB patients. ROC, receiver operating characteristic; ADRB3, adrenergic receptor $\beta 3$; ROCK2, Rho-related kinase 2; GEF, guanine nucleotide exchange factor; $\mathrm{OAB}$, overactive bladder.

Table 3. ROC curve values of ADRB3, ROCK2, GEF, and their combinations

\begin{tabular}{lcccc}
\hline Variable & AUC $(95 \%$ CI $)$ & P-value & Sensitivity \% & Specificity \% \\
\hline ADRB3 & $0.543(0.420-0.667)$ & 0.445 & 94.3 & 43.1 \\
ROCK2 & $0.651(0.535-0.767)$ & $0.008^{*}$ & 84.9 & 58.8 \\
GEF & $0.451(0.336-0.566)$ & 0.389 & 77.4 & 37.3 \\
ADRB3+ROCK2 c. & $0.700(0.602-0.798)$ & $<0.001^{\star}$ & 69.0 & 70.4 \\
ADRB3+GEF c. & $0.523(0.406-0.639)$ & 0.686 & 98.1 & 25.0 \\
ROCK2+GEF c. & $0.755(0.661-0.849)$ & $<0.001^{*}$ & 69.8 & 79.2 \\
ADRB3+ROCK2+GEF c. & $0.752(0.652-0.847)$ & $<0.001^{\star}$ & 64.2 & 88.2
\end{tabular}

ROC, receiver operating characteristic; ADRB3, adrenergic receptor $\beta 3$; ROCK2, Rho-related kinase 2; GEF, guanine nucleotide exchange factor; AUC, area under the curve; $\mathrm{CI}$, confidence interval; c, combination.

${ }^{\star} \mathrm{P}<0.05$, statistically significant difference. 
of 0.70 . The potential relationship of the ROCK2 + GEF combination was higher, with an AUC of 0.755 and sensitivity and specificity of $69.8 \%$ and $79.2 \%$ (95\% CI, 0.661-0.849; P < 0.001), respectively. The sensitivity and specificity of the ADRB3+ ROCK2 + GEF combination were $64.2 \%$ and $88.2 \%$ (95\% CI, 0.652-0.847; $\mathrm{P}<0.001$ ), respectively, with an AUC of 0.752 (Table 3). The ROC curves of the serum ADRB3 + GEF combination showed no significant results. The ROC curve values of all 3 parameters are presented in Table 3, and the ROC curves for the combinations are shown in Fig. 1B.

\section{DISCUSSION}

According to the results, the expression levels of ROCK2, which exerts effects on the cholinergic pathway, were upregulated significantly in $\mathrm{OAB}$ patients, while alterations in the levels of ADRB3 and GEF were non-significant. The relationships of these 3 markers with OAB had not been examined and reported previously. Previous studies by the current study authors examined the miRNAs that target and inhibit the function of genes encoding these 3 proteins, and significant dysregulation was found in the expression levels of these miRNAs. The diagnostic performance of the miRNAs was also seen to be sufficiently high. Therefore, it was concluded that alterations in miRNA levels, which alter the levels and function of ADRB3, ROCK2, and GEF in OAB patients, can be used as powerful biomarkers to help understand the pathophysiology of this condition and suggest new treatment options [14]. In another study, also by the current authors, the relationships of singlenucleotide polymorphisms in the 3 genes encoding ADRB3, ROCK2, and GEF with OAB were examined. Polymorphisms in these 3 genes were shown in $\mathrm{OAB}$ patients, but the relationship with OAB clinical status was not established [15]. The current study was another step in investigating the roles of these proteins in $\mathrm{OAB}$ pathophysiology.

Elevated levels of ROCK2, which functions in the cholinergic pathway, may result in $\mathrm{OAB}$ symptoms as a result of increased contraction via increased ROCK2 activation in the bladder smooth muscle. In support of this finding, in our previous study, the expression of miR-139-5p, which is thought to inhibit ROCK2 synthesis by targeting the gene responsible for ROCK2, was found to decrease in patients with $\mathrm{OAB}$ [14]. In the current study, ROCK2 was also seen to have a significant potential relationship in the ROC curves (AUC $=0.651$ ). This means that ROCK2 is a significant factor for diagnosing OAB patients. The dysregulation of ROCK2 alone and in combination with GEF or ADRB3 has good potential to support the OAB diagnosis. The ROCK2 + GEF combination had the highest potential relationship, with an AUC of 0.755 , indicating satisfactory predictive power. The ADRB3 + ROCK2 + GEF combination and the $\mathrm{ADRB} 3+\mathrm{ROCK} 2$ combination also had a substantial ability to differentiate patients from healthy controls, with AUCs of 0.752 and 0.700 , respectively. For the $\mathrm{OAB}$ symptom score, which is the most commonly used method to diagnose $\mathrm{OAB}$, Chuang et al. [16] and Arribillaga et al. [17] reported AUC values of 0.842 and 0.78 , respectively. Therefore, ROC analysis suggested that ROCK2, especially in combination with GEF or ADRB3, could act as an auxiliary biomarker for the diagnosis of OAB. Nerve growth factor [18] and brain-derived neurotrophic factor [19] have also both been proposed as potentially useful biomarkers for OAB patients. However, the potential of a relationship between urinary neurotrophins and the diagnosis of $O A B$ has not yet been clarified. Therefore, there is still a need to find reliable, noninvasive biomarkers to understand the pathophysiological pathways involved in $\mathrm{OAB}$ and to evaluate $\mathrm{OAB}$ patients more objectively.

Contrary to expectations, patients showed lower GEF levels than controls, but the difference was not significant. Similar to this finding, in our previous study, the expression of miR-92a$3 p$, which is thought to inhibit GEF synthesis by targeting the gene responsible for GEF, was elevated in patients with $\mathrm{OAB}$ [14]. This observation supports the finding of lower GEF levels in $\mathrm{OAB}$ patients, and these data suggest that different factors may affect the regulation of ROCK2 in the detrusor muscle contraction mechanism in OAB. This can be explained by the presence of unknown molecular mechanisms, the effects of which are not clear. More extensive studies on factors in the adrenergic and cholinergic systems are needed to explain their effects in $\mathrm{OAB}$ patients. Further studies can clarify the molecular mechanisms related to these genes in $\mathrm{OAB}$ patients.

One of the main limitations of this study is that the analyses were performed only with blood samples. The results could be supported by analyses of tissue and urine samples. Intravesical specimens were also not examined. Although wide variation between serum levels seems to be a limitation, this is inherent in protein analytes whose serum levels are measured in such small units.

In conclusion, ROCK2 and its combination with GEF and ADRB3 showed a strong potential relationship for discriminating $\mathrm{OAB}$ patients from healthy controls, but there was no cor- 
relation between the levels of these proteins and OAB-V8 symptom scores indicating the clinical severity of the disease. Therefore, it would not be straightforward to predict the prognosis of patients based on these protein levels. Patients' posttreatment protein levels could be measured to better assess this relationship. Although there were no correlations between the severity of $\mathrm{OAB}$ symptoms and the levels of ROCK2, ADRB3, and GEF, the findings of this study nonetheless suggest that ROCK2 alone, and particularly in combination with GEF or $\mathrm{ADRB} 3$, can serve as an auxiliary biomarker for the management of $\mathrm{OAB}$ in terms of patients' diagnosis and personalized treatment options. If ROCK2, ADRB3, and GEF levels can be analyzed in patients, patients with high ROCK2 and GEF levels could benefit more effectively from antimuscarinic therapy, and patients with low ADRB3 levels could benefit more effectively from beta-adrenergic therapy. Treatment success can be increased by patient-specific treatment. Furthermore, the significant elevation of ROCK2 levels in OAB patients may suggest its use as a treatment target and provide new perspectives for the development of pharmacological agents.

\section{AUTHOR CONTRIBUTION STATEMENT}

- Conceptualization: $E F, Z A, H A$

- Data curation: $E F, Z A, H A$

- Formal analysis: $E F, H A$

- Funding acquisition: $H A$

- Methodology: EF, ZA, $H A$

- Project administration: EF, ZA, HA

- Visualization: $E F, H A$

- Writing-original draft: $E F, Z A, H A$

-Writing-review \& editing: $E F, Z A, H A$

\section{ORCID}

Elif Firat

0000-0001-8394-3810

Zafer Aybek

0000-0002-4617-8854

Hülya Aybek

0000-0002-0635-4251

\section{REFERENCES}

1. Abrams P, Cardozo L, Fall M, Griffiths D, Rosier P, Ulmsten U, et al. The standardisation of terminology of lower urinary tract function: report from the Standardisation Sub-committee of the International Continence Society. Neurourol Urodyn 2002;21:167-78.
2. Antunes-Lopes T, Carvalho-Barros S, Cruz CD, Cruz F, MartinsSilva C. Biomarkers in overactive bladder: a new objective and noninvasive tool? Adv Urol 2011;2011:382431.

3. Meng E, Lin WY, Lee WC, Chuang YC. Pathophysiology of overactive bladder. Low Urin Tract Symptoms 2012;4 Suppl 1:48-55.

4. Yoshimura N, Chancellor MB. Neurophysiology of lower urinary tract function and dysfunction. Rev Urol 2003;5 Suppl 8(Suppl 8):S3-10.

5. Puetz S, Lubomirov LT, Pfitzer G. Regulation of smooth muscle contraction by small GTPases. Physiology (Bethesda) 2009;24:34256.

6. Kimura K, Ito M, Amano M, Chihara K, Fukata Y, Nakafuku M, et al. Regulation of myosin phosphatase by Rho and Rho-associated kinase (Rho-kinase). Science 1996;273:245-8.

7. Amano M, Ito M, Kimura K, Fukata Y, Chihara K, Nakano T, et al. Phosphorylation and activation of myosin by Rho-associated kinase (Rho-kinase). J Biol Chem 1996;271:20246-9.

8. Sharma P, Roy K. ROCK-2-selective targeting and its therapeutic outcomes. Drug Discov Today 2020;25:446-55.

9. Ferreira CE, Fonseca AM, Silva ID, Girão MJ, Sartori MG, Castro RA. The relationship between the Trp 64 Arg polymorphism of the beta 3-adrenoceptor gene and idiopathic overactive bladder. Am J Obstet Gynecol 2011;205:82.e10-4.

10. Gormley EA, Lightner DJ, Faraday M, Vasavada SP; American Urological Association; Society of Urodynamics, Female Pelvic Medicine. Diagnosis and treatment of overactive bladder (nonneurogenic) in adults: AUA/SUFU guideline amendment. J Urol 2015;193:1572-80.

11. Chapple CR. Muscarinic receptor antagonists in the treatment of overactive bladder. Urology 2000;55(5A Suppl):33-46.

12. Huang CK, Lin CC, Lin AT. Effectiveness of antimuscarinics and a beta-3 adrenoceptor agonist in patients with overactive bladder in a real-world setting. Sci Rep 2020;10:11355.

13. Tarcan T, Mangır N, Ozgur O, Akbal C. OAB-V8 Asırı aktif mesane sorgulama formu validasyon calısmasi. Uroloji Bulteni 2012; 21:113-6.

14. Firat E, Aybek Z, Akgün Ş, Küçüker K, Akça H, Aybek H. Exploring biomarkers in the overactive bladder: Alterations in miRNA levels of a panel of genes in patients with OAB. Neurourol Urodyn 2019;38:1571-8.

15. Firat E, Aybek Z, Akgün Ş, Küçüker K, Akça H, Aybek H. Relation of ADRB3, GEF, ROCK2 gene polymorphisms to clinical findings in overactive bladder. World J Urol 2020;38:2571-5.

16. Chuang FC, Hsiao SM, Kuo HC. The overactive bladder symptom score, International Prostate Symptom Score-storage subscore, and 
urgency severity score in patients with overactive bladder and hypersensitive bladder: which scoring system is best? Int Neurourol J 2018;22:99-106.

17. Arribillaga LC, Ledesma M, Montedoro A, Pisano F, Bengió RG. OAB score: a clinical model that predicts the probability of presenting overactive detrusor in the urodynamic study. Int Braz J Urol 2018;44:348-54.
18. Suh YS, Ko KJ, Kim TH, Lee HS, Sung HH, Cho WJ, et al. Potential biomarkers for diagnosis of overactive bladder patients: urinary nerve growth factor, prostaglandin $\mathrm{E} 2$, and adenosine triphosphate. Int Neurourol J 2017;21:171-7.

19. Wang LW, Han XM, Chen CH, Ma Y, Hai B. Urinary brain-derived neurotrophic factor: a potential biomarker for objective diagnosis of overactive bladder. Int Urol Nephrol 2014;46:341-7. 